\title{
Comparison of Satisfaction Levels between Conventional and Silicone-Adhesive Polyurethane Foam Materials in Patients with Skin Wounds
}

\author{
Hyun Jeong Ha (D), Jun Young Yang (D), Chan Woo Kim (D), Seong Heum Jeong (D), Euna Hwang (D) \\ Department of Plastic and Reconstructive Surgery, CHA Bundang Medical Center, CHA University School of Medicine, Seongnam, Korea
}

\begin{abstract}
Background: Polyurethane (PU) foam dressing materials have been widely used in commercial wound dressing applications. However, the repeated application of adhesive tapes to keep the foam dressings in place can result in minor injuries to the peri-wound skin. Silicone-adhesive PU foam dressing materials have been developed to prevent such injuries. In this study, the satisfaction levels between conventional and silicone-adhesive PU foams were assessed through a survey of patients and physicians.

Methods: A survey study of 140 patients with skin wounds was conducted in a single institution between July 2019 and May 2020 . The patients were first treated with either conventional PU foam or silicone-adhesive PU foam, after which they were asked to record their levels of pain, adhesiveness, waterproofness, and satisfaction. At the next visit, dressings of the other material were applied to their wounds, and the same assessment process was repeated at the next dressing change.

Results: The silicone-adhesive PU foam dressings demonstrably reduced the levels of dressing-related trauma and pain, compared to that of patients treated with conventional PU foam dressings. The silicone-adhesive PU foam dressings were also associated with substantially higher scores of satisfaction and waterproofness. In comparison, the mean adhesiveness score was superior in the group treated with conventional PU foam dressings, compared to that of the group treated with silicone-adhesive PU foam dressings.

Conclusion: Silicone-adhesive PU foam contributed to minimizing pain during dressing change and increasing patient's comfort. As a result, patients preferred dressing with silicone-adhesive PU foam over conventional PU foam.
\end{abstract}

Keywords: Wound dressing; Foam dressing; Polyurethane foam; Dressing materials

\section{Introduction}

Various types of dressing materials have been developed and commercialized over time. Since Winter [1] discovered the effectiveness of wet dressings in 1962, the concept of keeping the wound moist, rather than letting it dry, has proven to be effective in numerous clinical trials. Additionally, Junker et al. [2] confirmed that managing wounds in a wet environment enables delivery of antibacterial agents, along with other bioactive molecules such as growth factors. The addition of growth factors is likely to provide a regenerative wound microenvironment that promotes healing. On the other hand, if the surface of the wound is dry, the movement of epithelial cells is inhibited due to the collagen formed in the epidermal layer and the wound fluid expels the growth factors that accerelate angiogenesis and epithelialization [3]. Based on this understanding, dressing materials such as hydrogel [4], polyurethane (PU) foam [5,6], hydrocolloid [7], and hydrofiber [8], among others, have been developed to keep the wound moist.

In Korea, moist dressings with PU foam have been in wide use since 2002 [9]. PU

\section{Original Article}

Received: February 19, 2021

Revised: June 21, 2021

Accepted: June 21, 2021

\section{Corresponding author:}

Euna Hwang, M.D., Ph.D.

Department of Plastic and Reconstructive Surgery, CHA Bundang Medical Center, CHA University School of Medicine, 59 Yatap-ro, Bundang-gu, Seongnam 13496, Korea Tel: +82-31-780-5280

Fax: +82-31-780-5285

E-mail: pshwang70@gmail.com

This is an Open Access article distributed under the terms of the Creative Commons Attribution Non-Commercial License (https://creativecommons.org/licenses/by-nc/4.0/) which permits unrestricted non-commercial use, distribution, and reproduction in any medium, provided the original work is properly cited.

C) 2021 Korean Wound Management Society 
foam absorbs exudate from the wound and maintains adequate moisture on the wound surface. PU foam dressing material has the advantage of less adhesiveness to the wound, compared to simple gauze dressings, subsequently relieving pain during dressing change, and also provides a moist environment on the wound surface. Various PU foam dressing materials of different densities, particle sizes, and mixture qualities have been commercialized [10]. Previous studies have demonstrated that the use of PU foam dressing materials is effective for wound healing in a moist environment and is particularly useful in preventing dressing-related trauma and pain, in managing exuding wounds, and decreasing dressing discomfort [9]. In this study, the differences and satisfaction levels between conventional PU foams and silicone-adhesive PU foam were assessed through a survey of patients and physicians.

\section{Methods}

\section{Study design}

A single survey research was conducted in patients of different age groups with skin wounds who visited the plastic surgery department from July 2019 to May 2020. This study was approved by the Institutional Review Board of CHA Bundang Medical Center (IRB No. 2018-12-038). All the subjects provided written informed consent before participating in the study. The inclusion criteria were as follows: adult patients aged 20 to 80 years with the ability to take a survey to evaluate satisfaction; patients with acute skin lacerations or abrasions, burns, and postoperative incision wounds; and patients who provided written informed consent before participation in this study. The exclusion criteria were as follows: patients with dermatitis around the wound, immunocompromised patients, patients who were taking medications that may affect wound healing, patients with serious systemic conditions making them ineligible for wound healing studies, and patients who were unable to complete three clinical follow-ups. A total of 140 patients with skin wounds were identified. Forty patients were excluded, out of which 14 had dermatitis around the wound and 26 lacked clinical follow-up. After applying the exclusion criteria, a total of 100 patients were enrolled in this study.

We prepared two types of $\mathrm{PU}$ foam dressing materials for this study. One was a conventional PU foam (Meditouch; Ildong Pharmaceutical Co., Ltd., Seoul, Korea) without an adhesive border and the other type was a silicone-adhesive PU foam (Meditouch Border; Ildong Pharmaceutical Co., Ltd.) (Fig. 1).
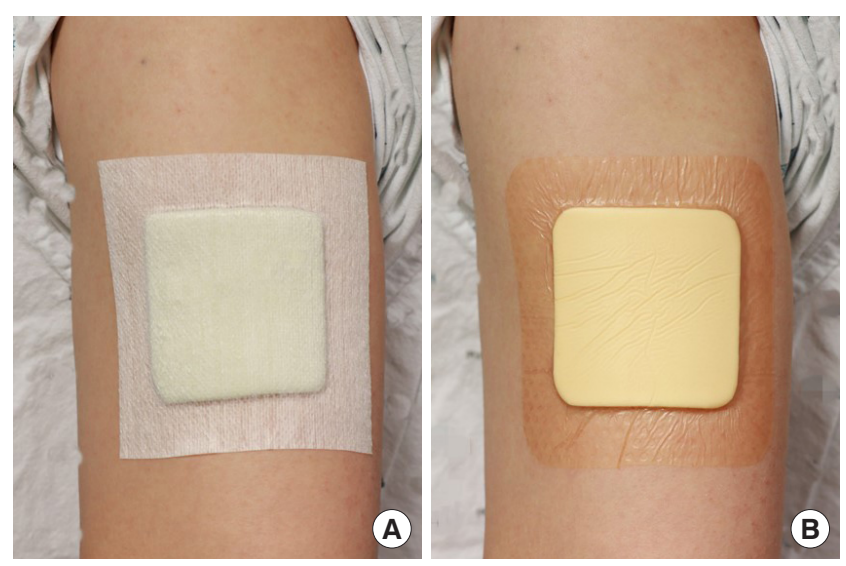

Fig. 1. Two types of polyurethane (PU) foam dressing materials. (A) Conventional and (B) silicone-adhesive PU foam dressing.

\section{Wound dressing}

The wound was cleaned with saline-soaked sponge and an antibiotic ointment (ofloxacin-Tarivid ophthalmic ointment; Santen Pharm., Osaka, Japan) was applied to the wound, after which it was covered with a randomly allocated PU foam dressing material. In the conventional PU foam group, the PU foam was trimmed to fit the size of the wound and was held in place using self-adhesive fabric tape (Micropore; $3 \mathrm{M} \mathrm{Co.,} \mathrm{Ma-}$ plewood, MN, USA). In the silicone-adhesive PU foam group, an adequately-sized silicone-adhesive PU foam was chosen and applied to the wound. Because the PU foam is typically used with self-adhesive fabric tape, for the purpose of this study, we hypothesized that the conventional PU foam and self-adhesive fabric tape were a single dressing material.

\section{Survey protocol}

The selected 100 patients were allocated into two groups (50 patients in each group) according to a random number table. One group was treated with the silicone-adhesive PU foam at the first visit, followed by a treatment with conventional PU foam at the second visit. In contrast, the other group was treated with the same method but in reverse order. The same protocol was applied for all the cases. Three clinical follow-ups were performed with 2-3 day intervals. At the first visit, the severity, size and location of the wound were assessed by physicians (CWK and JYY) in the outpatient clinic. They evaluated the wound status in terms of amount of exudate, injury level and infection signs, and the skin condition around the wound (erythema, rash, abrasion) during follow-up period. The duration of dressing change at every visit was also noted and these times were reflected in the score. 
The patients recorded answers to questions on adhesiveness, waterproofness, and pain level during and after dressing removal, rating the pain on a scale of $0-10(0=$ no pain; $10=$ unbearable pain) at the second visit. This visual analog scale has been validated for measuring pain severity in patients with wounds [11]. At the next visit, the dressing material was replaced with the alternate type. In the last follow-up (i.e., the third visit), adhesiveness, waterproofness, and pain levels were measured and recorded again. The waterproofness was evaluated by patients in terms of the degree of liquid absorption into the PU foam after light routine cleansing. The satisfaction of physicians and patients regarding the two types of $\mathrm{PU}$ foam dressing materials was finally surveyed on the third visit (Fig. 2).

Hospital Patient Satisfaction Survey: Outpatient Office

Please indicate your level of agreement with each of the following statements.

1. Choose a number from 0 to 10 that best describes your pain

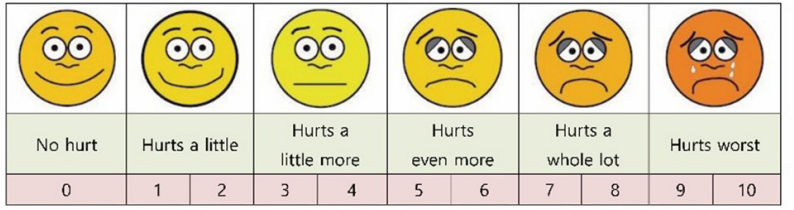

2. How satisfied are you overall with the dressing material you received?

\begin{tabular}{|c|c|c|c|c|c|}
\hline \multirow{2}{*}{ Satisfaction } & $\begin{array}{c}\text { Very } \\
\text { dissatisfied }\end{array}$ & Dissatisfied & $\begin{array}{c}\text { Neither } \\
\text { satisfied nor } \\
\text { dissatisfied }\end{array}$ & Satisfied & Very satisfied \\
\cline { 2 - 6 } & 1 & 2 & 3 & 4 & 5 \\
\hline
\end{tabular}

3. How satisfied are you with the adhesion level of the dressing material you received?

\begin{tabular}{|c|c|c|c|c|c|}
\hline \multirow{2}{*}{ Adhesiveness } & Very poor & Poor & Neutral & Good & Excellent \\
\cline { 2 - 6 } & 1 & 2 & 3 & 4 & 5 \\
\hline
\end{tabular}

4. How satisfied are you with the waterproofness level of the dressing material you received?

\begin{tabular}{|c|c|c|c|c|c|}
\hline \multirow{2}{*}{ Waterproofness } & Very poor & Poor & Neutral & Good & Excellent \\
\cline { 2 - 6 } & 1 & 2 & 3 & 4 & 5 \\
\hline
\end{tabular}

A

Hospital Physician Satisfaction Survey: Outpatient Office

Please indicate your level of agreement with each of the following statements.

1. Time taken to disinfect the affected area

: Time taken from removing the existing dressing to the end of treatment (min)

\begin{tabular}{|c|c|c|c|c|c|c|c|c|c|c|}
\hline $\begin{array}{c}\text { Time } \\
\text { (min) }\end{array}$ & $<5$ & $<4.5$ & $<4$ & $<3.5$ & $<3$ & $<2.5$ & $<2$ & $<1.5$ & $<1$ & $<0.5$ \\
\hline Score & 0.5 & 1 & 1.5 & 2 & 2.5 & 3 & 3.5 & 4 & 4.5 & 5 \\
\hline
\end{tabular}

B

Fig. 2. Satisfaction survey questionnaire. (A) Patient and (B) physician.

\section{Statistical analysis}

Continuous variables were presented as mean \pm standard deviation. Categorical variables were presented as frequency and proportions. The normality of distribution of continuous variables was tested by the Kolmogorov-Smirnov test (Table 1). Based on these test results, the sets of data for the two treatments were assumed to be not normally distributed. The Wilcoxon signed-rank test produced the exact $\mathrm{P}$-values for comparing the distribution of related samples. A value of $\mathrm{P}<0.05$ was considered significant. Statistical analysis was performed using SPSS version 25.0 (IBM Corp., Armonk, NY, USA).

\section{Results}

A total of 100 patients were enrolled in this study. Patient demographics are presented in Table 2. The patients presented with a variety of wound locations, including the head and neck, upper extremities, trunk, and lower extremities; wound types included burns, skin lacerations, and surgical wounds. The physicians checked the time taken when dressing with two types of dressing materials. The mean time score associated with the conventional PU foam dressing was higher than that of the silicone-adhesive PU foam dressing ( $1.5 \pm 0.6$ vs. $1.6 \pm 0.6, P=0.017)$. However, there was no statistical significance (Table 3). Meanwhile, we defined adhesiveness as an indicator showing how well it is attached to the wound area.

Pain scores associated with the conventional PU foam dressing, compared with those associated with the silicone-adhesive PU foam dressing, are presented in Fig. 3. The mean pain

Table 1 . Normality test

\begin{tabular}{lcccc}
\hline \multirow{2}{*}{ Test } & & \multicolumn{3}{c}{ Kolmogorov-Smirnov test $^{\mathrm{a})}$} \\
\cline { 3 - 5 } & & Statistics & Degree of freedom & P-value \\
\hline Time & A & 0.222 & 100 & 0.000 \\
Pain & B & 0.203 & 100 & 0.000 \\
& A & 0.273 & 100 & 0.000 \\
Satisfaction & B & 0.169 & 100 & 0.000 \\
& B & 0.309 & 100 & 0.000 \\
Adhesiveness & A & 0.222 & 100 & 0.000 \\
& B & 0.284 & 100 & 0.000 \\
Waterproofness & A & 0.289 & 100 & 0.000 \\
& B & 0.196 & 100 & 0.000 \\
& B & 100 & 0.000
\end{tabular}

A, silicone-adhesive polyurethane (PU) foam; B, conventional PU foam. a) Lilliefors significance correction. 
score in the conventional PU foam dressing was $2.3 \pm 1.8$. In comparison, a significantly lower mean pain score $(0.9 \pm 1.2$, $\mathrm{P}=0.000$ ) was reported in the silicone-adhesive PU foam dressing. Unlike the conventional PU foam dressing, the silicone-adhesive PU foam dressing was not associated with increased pain during dressing change.

The silicone-adhesive PU foam dressing showed better waterproofness than the conventional PU foam dressing ( $4.1 \pm 0.8$ vs. $3.1 \pm 1.0, \mathrm{P}=0.000)$. Furthermore, the mean satisfaction score in the silicone-adhesive PU foam dressing was much higher than that of the conventional PU foam dressing ( $4.0 \pm$ 0.7 vs. $3.4 \pm 0.9, \mathrm{P}=0.000$ ). This showed that the patients preferred the silicone-adhesive PU foam dressing to the other

Table 2. Patient demographics

\begin{tabular}{lc}
\hline Characteristic & Value \\
\hline No. of patients & $100(100)$ \\
Age (yr) & $48.15 \pm 15.54$ \\
$20-39$ & $30(30)$ \\
$40-59$ & $43(43)$ \\
$60-79$ & $27(27)$ \\
Sex & \\
Male & $56(56)$ \\
Female & $44(44)$ \\
Location & \\
Head \& neck & $47(47)$ \\
Upper extremities & $11(11)$ \\
Trunk & $27(27)$ \\
Lower extremities & $15(15)$ \\
Severity of the wound & \\
Mild & $24(24)$ \\
Moderate & $57(57)$ \\
Severe & $19(19)$ \\
\hline
\end{tabular}

Values are presented as number (\%) or mean \pm SD. dressing type. On the other hand, the mean adhesiveness score was lower in the silicone-adhesive PU foam dressing than in the conventional $\mathrm{PU}$ foam dressing ( $3.5 \pm 0.7$ vs. $4.3 \pm 0.6, \mathrm{P}=$ 0.000 ) (Fig. 4). Table 2 summarizes the results of the survey of patients treated with silicone-adhesive PU foam and conventional PU foam dressings.

\section{Discussion}

Standard wound management requires the right choice of dressing materials according to the status of the wound. An ideal dressing should be nontoxic, nonirritating, effectively antibacterial, able to protect the wound from the surrounding environment, and easy to apply and remove without causing skin injury [12]. Furthermore, it should maintain a moist environment and should be comfortable and acceptable to patients. Among the various types of dressing materials, $\mathrm{PU}$ foam dressings are commonly used to absorb exudates from the wound and shield the wound from the external environment. Many published clinical reports describe the effective-

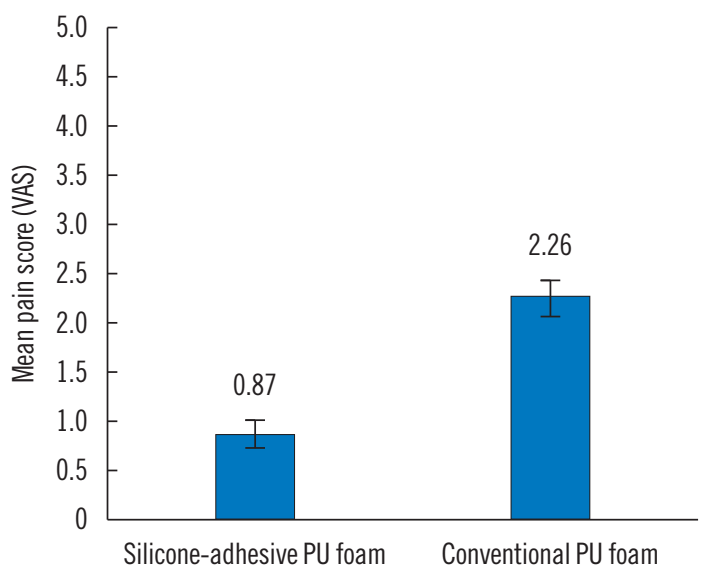

Fig. 3. Pain scores reported with the two types of dressing materials $(P<0.000)$. VAS, visual analog scale; PU, polyurethane.

Table 3. Summary of research outcomes involving the two types of dressing materials by an analysis of the survey

\begin{tabular}{lcccc}
\hline Variable & Silicone-adhesive PU foam & Conventional PU foam & $Z$ & P-value $^{\text {a) }}$ \\
\hline Time & $1.5 \pm 0.6(0.5-3.0)$ & $1.6 \pm 0.6(0.5-3.5)$ & -2.389 & 0.017 \\
Pain & $0.9 \pm 1.2(0-5)$ & $2.3 \pm 1.8(0-9)$ & -5.328 & 0.000 \\
Satisfaction & $4.0 \pm 0.7(2-5)$ & $3.4 \pm 0.9(1-5)$ & -4.584 & 0.000 \\
Adhesiveness & $3.5 \pm 0.7(2-5)$ & $4.3 \pm 0.6(2-5)$ & -6.731 & 0.000 \\
Waterproofness & $4.1 \pm 0.8(1-5)$ & $3.1 \pm 1.0(1-5)$ & -5.962 & 0.000 \\
\hline
\end{tabular}

Values are presented as mean \pm SD (range).

PU, polyurethane.

a)P-values were calculated by Wilcoxon signed-rank test. Based on positive ranks. 


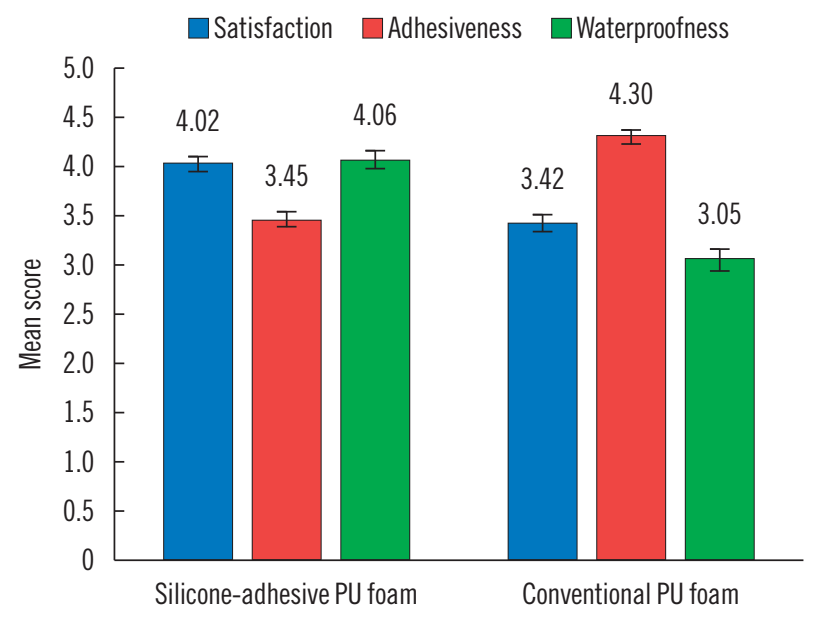

Fig. 4. Evaluation in the scores of satisfaction, adhesiveness, and waterproofness. PU, polyurethane.

ness of PU foam dressings in acute traumatic and surgical wounds. Clinical experience from these studies suggests that PU foam dressings are easy to apply, and are relatively less painful to remove. However, notwithstanding the functionality of PU foam dressings, they require secondary dressing materials such as self-adhesive fabric tapes to stay in place. While removing the tape, dressing-related injury of the surrounding skin frequently occurs for patients with fragile skin such as children, women and the elderly. Moreover, it is inconvenient and time-consuming to tailor the dressing material to the wound every time. One attempt to solve this problem is the use of silicone-adhesive dressing material, which consists of PU foam with a silicone film attached to one side that provides adherence to the skin. Silicone-adhesive PU foam is highly elastic and conformable to body contours and is suitable for use as a primary dressing. As it is also impermeable to water and minimizes dressing-related trauma to the surrounding skin when removed, silicone-adhesive PU foam allows for more convenient and safe application.

This study demonstrates that, compared to conventional PU foam dressings, silicone-adhesive PU foam dressings improve the satisfaction and convenience of both physicians and patients. In particular, statistically significant differences were observed in favor of silicone-adhesive PU foam dressings regarding the levels of wound-related pain, painless dressing removal and waterproofness. It also reduced dressing-related injuries to the surrounding skin. The waterproofing function of the silicone film of silicone-adhesive PU foam dressings can contribute to improving the patient's quality of life. Although the patients found the silicone-adhesive PU foam to be less adhesive than conventional PU foam, physicians applying the material in clinical settings reported satisfactory adhesiveness. The only disadvantage of silicone-adhesive PU foam is that it is inconvenient to apply to small curved surfaces such as the regions of the eyes, nose, and mouth.

In conclusion, we compared two types of commercially available PU foam dressing materials: conventional PU foam and silicone-adhesive PU foam. Our survey-based study provides insights into the key differences between dressing materials. Because silicone-adhesive PU foam produces less injuries to the peri-wound tissue during dressing change, it can be useful in patients with frail or sensitive skin, such as children, women, and the elderly. Overall, we found that patients preferred dressings with silicone-adhesive PU foam to those with conventional PU foam.

\section{Conflict of interest}

This work was supported by the Ildong Pharmaceutical Co., Ltd., the manufacturer of two types of polyurethane dressing materials used in the study. No other potential conflicts of interest relevant to this article are reported.

\section{ORCID iDs}

Hyun Jeong Ha

Jun Young Yang

Chan Woo Kim

Seong Heum Jeong

Euna Hwang

https://orcid.org/0000-0001-6039-4804

https://orcid.org/0000-0003-4995-9813

https://orcid.org/0000-0002-7441-8480

https://orcid.org/0000-0003-4407-2914

https://orcid.org/0000-0002-0077-3092

\section{References}

1. Winter GD. Formation of the scab and the rate of epithelization of superficial wounds in the skin of the young domestic pig. Nature 1962;193:293-4.

2. Junker JP, Kamel RA, Caterson EJ, et al. Clinical impact upon wound healing and inflammation in moist, wet, and dry environments. Adv Wound Care (New Rochelle) 2013; 2:348-56,

3. Kim WI, Kim CJ, Kim DY, et al. Fabrication and characterization of polyurethane foam for wound dressing. Polymer (Korea) 2010;34:442-9.

4. Vogt PM, Hauser J, Rossbach O, et al. Polyvinyl pyrrolidone-iodine liposome hydrogel improves epithelialization by combining moisture and antisepsis: a new concept in 
wound therapy. Wound Repair Regen 2001;9:116-22.

5. Di Benedetto G, Pierangeli M, Scalise A, et al. An improved tie-over dressing technique for skin grafts using a hydrocellular dressing. Plast Reconstr Surg 2000;106:507-9.

6. Williams C, Young T. Allevyn adhesive. Br J Nurs 1996;5: 691-3.

7. Hermans MH. HydroColloid dressing (Duoderm) for the treatment of superficial and deep partial thickness burns. Scand J Plast Reconstr Surg Hand Surg 1987;21:283-5.

8. Walker M, Hobot JA, Newman GR, et al. Scanning electron microscopic examination of bacterial immobilisation in a carboxymethyl cellulose (AQUACEL) and alginate dressings. Biomaterials 2003;24:883-90.
9. Park YO, Minn KW, Hur JP. The effect of Medifoam (hydrophilic polyurethane foam) dressing in split thickness skin graft donor site. J Korean Soc Plast Reconstr Surg 2002; 29:297-301.

10. Lee SM, Park IK, Kim YS, et al. Physical, morphological, and wound healing properties of a polyurethane foam-film dressing. Biomater Res 2016;20:15.

11. Freeman K, Smyth C, Dallam L, et al. Pain measurement scales: a comparison of the visual analogue and faces rating scales in measuring pressure ulcer pain. J Wound Ostomy Continence Nurs 2001;28:290-6.

12. Dhivya S, Padma VV, Santhini E. Wound dressings: a review. Biomedicine (Taipei) 2015;5:22. 